\title{
Arquitectura basada en contexto para el soporte del servicio de VoD
}

\author{
Gabriel E. Chanchí G. ${ }^{1}$, Wilmar Y. Campo M. ${ }^{2}$, Jose L. Arciniegas H. ${ }^{3}$ \\ gchanchi@unimayor.edu.co, wycampo@uniquindio.edu.co,jlarci@unicauca.edu.co \\ ${ }^{1}$ Institución Universitaria Colegio Mayor del Cauca, Cra. 7 \# 2-34, 1900o3, Popayán, Colombia. \\ ${ }^{2}$ Universidad del Quindío, Cra. 15 Cll 12 norte, 630004, Armenia, Colombia. \\ ${ }^{3}$ Universidad del Cauca, Campus Tulcán, 190003, Popayán, Colombia.
}

DOI: 10.17013/risti.29.55-71

\begin{abstract}
Resumen: A pesar de la amplia difusión del servicio de video bajo demanda (VoD), se destacan dos problemas principales a nivel funcional como son: permitir el acceso ágil al contenido multimedia y posibilitar el consumo adecuado de dicho contenido. Estos retos pueden ser abordados desde las diferentes dimensiones de la definición de contexto para servicios de telecomunicaciones (usuario, red, dispositivo, servicio), teniendo en cuenta tecnologías como los sistemas de recomendación basados en contexto en la dimensión de usuario y el streaming adaptativo DASH en la dimensión de red y del dispositivo. Así, este artículo propone una arquitectura basada en contexto para el servicio de VoD, la cual considera las diferentes dimensiones del contexto y es organizada en 4 vistas a saber: de negocio, de contexto, funcional y de implementación. Esta arquitectura pretende enriquecer la funcionalidad del servicio y contribuir con los dos retos principales del servicio de VoD.
\end{abstract}

Palabras-clave: Arquitectura; contexto; DASH; sistemas de recomendación; video bajo demanda.

\section{Context-based architecture for VoD service support}

\begin{abstract}
In spite of the wide diffusion of the service of video on demand (VoD), two main problems at functional level stand out: to allow the agile access to the multimedia content and to allow the adequate consumption of the contained content. These challenges can be addressed from the different dimensions of the context definition for telecommunications services (user, network, device, service), taking into account technologies such as context-based recommendation systems in the user dimension and adaptive streaming (DASH) in the network and device dimension. Thus, in this paper we propose a context-based architecture for the VoD service, which considers the different dimensions of the context and is organized into 4 views to know: business, context, functional and implementation. This architecture aims to enrich the functionality of the service and contribute to the two main challenges of the VoD service.
\end{abstract}

Keywords: Architecture; context; DASH; recommender systems; video on demand. 


\section{Introducción}

En los últimos años, los proveedores de servicios de Internet han incrementado las velocidades de acceso a sus suscriptores. Estas mejoras en el ancho de banda han propiciado el aprovechamiento de estas redes para el consumo de servicios basados en transmisión de audio y vídeo, como es el caso del streaming (Campo, Arciniegas, Garcia, \& Melendi, 2010). A través del uso del streaming no es necesario que el cliente descargue toda la información para consumirla, ya que esta se va almacenando en un búfer y se ejecuta al mismo tiempo que se trasmite (Mack, 2002).

Uno de los servicios cuya transmisión está soportada en streaming es IPTV, definida por la ITU-T como el envío de servicios multimedia de alta calidad a través de redes IP de banda ancha usando protocolos de streaming (ITU-T, 2008). Dentro de los servicios de distribución de contenidos multimedia, se destaca el servicio de video bajo demanda VoD (Pripuzic, et al., 2013), el cual consiste en una aplicación que espera, procesa y sirve peticiones de uno, o varios clientes. Para facilitar el acceso a los contenidos multimedia, este servicio provee un catálogo de contenidos, a través del cual el televidente navega, escoge y reproduce los contenidos multimedia (Campo, et al., 2010).

A pesar de las ventajas del servicio de $\mathrm{VoD}$, existen un conjunto de problemas que dificultan la interacción del usuario, como son: el crecimiento de los catálogos de contenidos y los limitados métodos de entrada (control remoto) lo que incrementa el tiempo empleado por un usuario al navegar por estos catálogos, además se tiene la fluctuación del ancho de banda en el momento de reproducir el contenido multimedia y las diferentes características de los dispositivos que acceden al servicio (colores, codecs, resolución) (Dabrowski, Gromada, \& Moustafa, 2012) (Song, Moustafa, \& Afifi, 2011) (Turrin \& Cremonesis, 2010). Las anteriores problemáticas pueden clasificarse dentro de dos retos principales para el servicio de VoD: facilitar el acceso ágil y permitir consumo adecuado del contenido multimedia en el entorno de televisión.

Con respecto al primer reto del servicio de VoD, surgen los sistemas de recomendación (Dabrowski, et al., 2012) (Song, et al., 2011), los cuales son agentes encargados de identificar las preferencias de un usuario, para guiarlo de forma personalizada en el proceso de escogencia de un ítem a partir de muchas opciones (Jannach, Zanker, Felfernig, \& Friedrich, 2010) (Yager, 2003). De acuerdo con la literatura, estos se dividen en 2 enfoques clásicos (Jannach, et al., 2010) (Yager, 2003) (Porcel, López-Herrera, \& Herrera, 2009) (Porcel, Moreno, \& Herrera, 2009) (Porcel, Tejeda, Martínez, \& Herrera, 2012) (Zanker, Klagenfurt, Jannach, Gordea, \& Jessenitschnig, 2007) (Melville, Mooney, \& Nagarajan, 2002): los basados en filtros de contenidos y los basados en filtros colaborativos, los cuales se encargan de recomendar contenidos de acuerdo al perfil del usuario o a partir de un conjunto de usuarios con un perfil similar. Estos dos enfoques tienen como dificultad común el problema de arranque en frío (Turrin \& Cremonesis, 2010) (Jannach, et al., 2010) que implica que el sistema no tenga contenidos para recomendar en estados preliminares. Como alternativa a este problema, se destacan los sistemas de recomendación basados en contexto, los cuales hacen uso de variables del contexto de usuario para inferir información de interés en estados preliminares.

Con respecto al segundo reto del servicio de $\mathrm{VoD}$, surge la técnica conocida como streaming adaptativo, especificada por el 3GPP como Adaptive HTTP Streaming (AHS) 
(Arzelier, 2017), la cual consiste en cortar un archivo multimedia en segmentos de igual duración que pueden ser codificados en diferentes resoluciones y tasas de bits, que son suministrados a un servidor web para descargarse vía HTTP. Con el fin de establecer la relación entre tasas de bits, segmentos y el orden de los mismos, AHS hace uso del archivo Media Presentation Description (MPD), el cual contiene una descripción formal sobre una colección de datos, que representan las características técnicas del contenido (ISO/IEC 23009-1:2012, 2014).

La industria ha desplegado varias soluciones propietarias de streaming adaptativo, como las descritas en (Zambelli, 2009) (Adobe, 2012). Dichas soluciones tienen como inconveniente el empleo de técnicas propias de segmentación, generación de secuencia de tiempo y creación de formatos MPD (Ortiz, Chanchí, Arciniegas, \& Duran, 2017), lo cual impide la compatibilidad a la de construir o consumir servicios de video. Para lograr la entrega eficiente de contenidos utilizando HTTP en sus diferentes formas: adaptativa, progresiva, streaming/descarga, así como garantizar la interoperabilidad entre las soluciones propietarias, MPEG (Moving Picture Expert Group) desarrolló Dynamic Adaptive Streaming over HTTP (DASH) (ISO/IEC 23009-1:2012, 2014). Según (Casín, 2013), DASH se puede definir como un sistema por el cual se proporcionan formatos, que habilitan la entrega eficiente y de alta calidad de servicios de streaming, permitiendo las siguientes ventajas (Gambín, 2012): reutilización de la tecnología existente, despliegue sobre HTTP, menor tiempo de arranque, selección de la calidad en función de la red y las características del dispositivo, cambios entre calidades transparentes al usuario y coexistencia con tecnologías propietarias.

Las alternativas a los retos del servicio de VoD, pueden enmarcarse en la definición del contexto en el ámbito de las telecomunicaciones, el cual es entendido como cualquier información, que puede usarse para caracterizar la situación de una entidad relacionada con el servicio, siendo una entidad cualquier elemento que incide en la interacción entre el usuario y el servicio (Dabrowski, et al., 2012). Así, en el ámbito de IPTV el contexto tiene cuatro dimensiones: usuario, dispositivo, red y servicio, cada una de las cuales tiene asociadas un conjunto de variables que pueden incidir en la interacción entre el usuario y el servicio (Dabrowski, et al., 2012) (Thawani, Gopalan, \& Sridhar, 2004) (Chen \& Kotz, 2000). En el caso del primer reto correspondiente a facilitar el acceso ágil, variables del contexto de usuario pueden ser utilizadas para inferir información como el estado de ánimo, la actividad diaria, que contribuyan con el problema del arranque en frío de los sistemas de recomendación clásicos, mejorando así la precisión de los mismos. Con respecto al segundo reto, variables como el ancho de banda y la resolución del dispositivo de acceso pueden considerarse a través de DASH, con el fin de mejorar los problemas de fluctuación de ancho de banda y acceso al servicio desde diferentes dispositivos.

Este artículo propone una arquitectura basada en contexto para abordar los dos principales retos del servicio de VoD. Esta arquitectura incluye las diferentes dimensiones del contexto (usuario, red, dispositivo y servicio) haciendo uso de sistemas de recomendación basados en contexto y streaming DASH. A través de los sistemas de recomendación basados en contexto se pretende considerar el contexto de usuario, aportando así al reto de facilitar el acceso ágil al contenido multimedia. De igual forma, mediante el DASH se busca abordar el contexto de red y dispositivo, aportando al reto de permitir el consumo adecuado de contenido. 
El resto del artículo está organizado de la siguiente forma: en la sección 2 se describen los trabajos relacionados con la presente investigación. En la sección 3 se muestra la propuesta arquitectónica abordada en este artículo. En la sección 4 se describe un servicio de VoD implementado a partir de la arquitectura propuesta. Finalmente, en la sección 5 se presentan las conclusiones y trabajos futuros.

\section{Trabajos relacionados}

En esta sección se describen un conjunto de trabajos relacionados con la presente investigación, los cuales se han organizado en tres temáticas: arquitecturas basadas en contexto, sistemas de recomendación basados en contexto, y entornos de streaming adaptativo DASH.

\subsection{Arquitecturas basadas en contexto}

En (da Silva, Alves, \& Bressan, 2009) los autores presentan una arquitectura para el soporte de recomendaciones personalizadas basadas en contexto, sobre entornos de Televisión Digital Interactiva (TDi) soportados en los mediadores DVB-MHP y GINGA con el objeto de mejorar el servicio de guía interactiva EPG. En (Dabrowski, et al., 2012) se presenta una arquitectura para el soporte de servicios personalizados en el ámbito de IPTV, considerando características del contexto del usuario, del dispositivo de acceso, de la red y de los servicios interactivos. La arquitectura busca responder a la necesidad de servicios personalizados, como una alternativa al crecimiento de los catálogos de contenidos multimedia. En (Thawani, et al., 2004) se presenta una arquitectura para la selección e inserción de anuncios publicitarios basados en el contexto, sobre escenarios de TDi. El objetivo de la arquitectura es permitir la personalización de los anuncios, de acuerdo a las preferencias de usuario, estos últimos obtenidos mediante reglas de inferencia aplicadas a las variables del contexto, aportando importantes conceptos en cuanto al manejo de reglas de inferencia sobre el contexto. En (Song, et al., 2011) los autores presentan una arquitectura para servicios personalizados de IPTV, la cual se enmarcan dentro del dominio NGN IPTV. La arquitectura planteada busca responder a la necesidad de filtrar el contenido y los servicios adecuados de acuerdo al perfil del usuario.

Los trabajos anteriores no han sido desplegados en escenarios móviles, de este modo, estos trabajos consideran como información del contexto: la hora, la distancia al STB (Set Top Box) y el historial del usuario (Dabrowski, et al., 2012) (Song, et al., 2011) (da Silva, et al., 2009), lo cual limita la precisión de los contenidos presentados por los sistemas de recomendación vinculados a la arquitectura. Así, estas arquitecturas, no consideran variables de tipo fisiológico en el contexto. Con respecto a la dimensión de red, esta es abordada en (Dabrowski, et al., 2012) (Song, et al., 2011), a través de un conjunto de sensores software distribuidos sobre la red, de tal forma que en ninguno de los 2 casos se hace uso de DASH. En lo que se refiere al contexto desde la dimensión del dispositivo de acceso, tanto en (Dabrowski, et al., 2012) como en (Song, et al., 2011) se tienen en cuenta características básicas del STB, con el fin de determinar los codecs adecuados para la reproducción del contenido multimedia. 


\subsection{Sistemas de recomendación basados en contexto}

En (Wang, Rosenblum, \& Wang, 2012) se presenta un sistema de recomendaciones de contenidos musicales para entornos móviles basado en filtros colaborativos, el cual hace uso de información obtenida a partir del contexto (clima, posición, hora), que es usada para filtrar el contenido multimedia a recomendar. En (Park, Yoo, \& Sung-Bae, 2006) se propone un sistema de recomendación híbrido para contenidos multimedia musicales, el cual tiene en cuenta información del contexto de usuario, obtenida a partir de sensores y diversas fuentes de internet. Dentro de las variables obtenidas se encuentran: temperatura, humedad, ruido, clima, hora y estación. En (Rho, Han, \& Hwang, 2009) se presenta un sistema de recomendaciones basado en contexto para contenidos multimedia musicales, teniendo en cuenta el estado de ánimo del usuario.

En (Su, Yeh, Yu, \& Tseng, 2010) se propone un sistema de recomendaciones ubicuo, para contenidos multimedia musicales, el cual tiene en cuenta información del contexto de usuario y de los demás usuarios del servicio de música bajo demanda. En (Cunningham, Caulder, \& Grout, 2008) se propone un sistema basado en contexto, para la generación de listas automáticas de reproducción de contenidos multimedia musicales. La información del contexto es obtenida a partir de dos fuentes principales: el usuario y el entorno donde el usuario escucha la música. En (Pripuzic, et al., 2013) se plantea un sistema de recomendaciones para contenidos multimedia, del servicio de video bajo demanda (VoD) de IPTV. En (Zhang \& Zheng, 2005) se presenta un sistema de recomendaciones, para contenidos multimedia de televisión digital, los cuales son descritos mediante el esquema de metadatos TV-Anytime. El sistema de recomendaciones desarrollado en este trabajo fue evaluado bajo una arquitectura del mediador DVB MHP. En (Turrin \& Cremonesis, 2010) se describen los problemas de la TDi relacionados con el número de canales existentes y el tiempo del zapping. Además, se plantean los sistemas de recomendación de contenidos como posible solución a estas falencias, proponiendo una arquitectura base para el diseño estos sistemas.

En (Melville, et al., 2002) se presentan las ventajas y desventajas de los sistemas de recomendación basados en las dos aproximaciones clásicas: filtros de contenidos y filtros colaborativos. Los autores diseñan e implementan un recomendador híbrido, el cual combina la técnica de predicción probabilística del clasificador de Naive Bayes, con el algoritmo del vecindario usado en filtros colaborativo. En (Asanov, 2011) se exponen las técnicas, métodos, algoritmos y problemáticas, consideradas en la construcción de sistemas de recomendación tradicionales y modernos. En (Chanchí, Campo, Amaya, \& Arciniegas, 2011) los autores proponen un esquema para el consumo de servicios interactivos de la Web 2.0 (foros, micro-blog, chats), sobre diversos entornos como la televisión digital terrestre la televisión móvil e IPTV.

Los trabajos anteriores presentan tanto aproximaciones clásicas de sistemas de recomendación de contenidos de televisión (Pripuzic, et al., 2013) (Turrin \& Cremonesis, 2010) (Melville, et al., 2002) (Zhang \& Zheng, 2005) (Asanov, 2011), como recomendadores basados en contexto para contenidos musicales (Wang, et al., 2012) (Park, et al., 2006) (Su, et al., 2010). Con respecto al primer caso, una de las limitaciones para no vincular el contexto a las recomendaciones es la dificultad para acceder a variables externas, por no ser desplegados los sistemas propuestos en estos 
trabajos en escenarios móviles. En el segundo caso, los recomendadores considerados no están enmarcados en entornos de televisión, ni trabajan con contenidos multimedia de video. Así mismo, estos sistemas de recomendación no consideran dentro del contexto variables asociadas al espacio corporal del usuario o también conocidas como variables fisiológicas (ritmo cardiaco, conductividad de la piel, expresión facial, entre otras), las cuales pueden reflejar información más precisa acerca de las necesidades emocionales de un usuario. A pesar de esto, los métodos usados en cuanto a clasificación e inferencia de contenidos multimedia a partir de variables locales (ubicación, hora, temperatura, etc.), pueden ser tenidos en cuenta en el manejo de variables del contexto para contenidos multimedia de video sobre IPTV. En ninguno de estos trabajos, el entorno usado para la difusión de contenidos multimedia tiene en cuenta las características de la red ni las de los dispositivos de acceso.

\subsection{Entornos de streaming adaptativo DASH}

En (Li \& Bouazizi, 2013) se proporcionan bases esenciales para conocer las diversas capacidades y la flexibilidad que ofrece el estándar MPEG-DASH para vincular servicios adicionales. En (Muller, et al., 2013) los autores explican cómo los proveedores de contenidos multimedia adaptativo: Netflix y YouTube no despliegan su propia arquitectura de streaming, pero ofrecen sus servicios OTT (Over-The-Top), basándose en el uso de la infraestructura de HTTP. Este artículo describe un conjunto de herramientas del lado del servidor y del lado del cliente, para la codificación y recepción de contenidos multimedia adaptativos, soportados en el estándar MPEG-DASH. En (Timmerer \& Griwodz, 2012) se presenta una guía sobre el despliegue de la tecnología de streaming adaptativo a través de HTTP, teniendo en cuenta la creación de contenidos, la entrega, el consumo y la evaluación de los mismos, con respecto a las soluciones propietarias.

Los anteriores trabajos presentan un conjunto de herramientas para la codificación, difusión y consumo de streaming adaptativo, basado en DASH. Sin embargo, ninguno de estos trabajos plantea un servicio de video bajo demanda soportado en la tecnología de streaming adaptativo DASH.

\section{Propuesta de la arquitectura}

Esta sección presenta la propuesta de la arquitectura para el servicio de VoD, la cual es descrita desde cuatro vistas a saber: vista de contexto, vista de negocio, vista funcional y vista de implementación. Esta arquitectura fue escogida para ser presentada en vistas tomando las ideas del modelo de vistas arquitectónicas 4+1 de Kruchten (Kruchten, 1995), definiendo y personalizando las vistas más representativas para el servicio de VoD. La arquitectura se diseñó a partir de un conjunto de consideraciones extraídas de la revisión literaria, ver figura 1.

La arquitectura para el servicio de VoD en sus vistas debe considerar la definición del contexto en sus diferentes dimensiones, con el fin de enriquecer y mejorar la percepción del usuario con respecto al servicio. Así, desde la dimensión de red y dispositivo se debe aportar al reto de permitir el consumo adecuado del contenido, usando el estándar DASH, considerando que este estándar permite la adaptación de los contenidos al ancho de banda de la red y a las características de los contenidos. Del mismo modo, desde la 


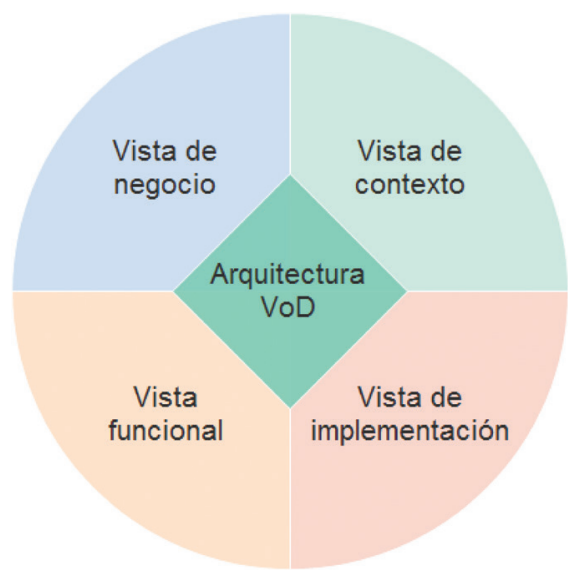

Figura 1 - Vistas de la arquitectura

dimensión de usuario se debe aportar al reto de garantizar el acceso ágil al contenido multimedia, considerando los sistemas de recomendación basados en contexto, dado que estos contribuyen a mejorar el problema de arranque en frío y la precisión de las recomendaciones.

\subsection{Vista del negócio}

En el presente trabajo, la vinculación del contexto al servicio de VoD en sus diferentes dimensiones: usuario, red y dispositivo implica un cambio en las tareas realizadas en los eslabones de la cadena de valor tradicional del servicio, específicamente en los dominios de proveedor de servicio y consumidor. En la figura 2, se presentan cada una de las tareas propuestas para cada uno de los eslabones de la cadena de valor del servicio, incluyendo las del servicio tradicional de VoD.

En el dominio del proveedor de contenido se conservan las tareas de producción y edición del contenido, así como la generación de metadatos. En el dominio del proveedor del servicio se incluyen las tareas de generación del contenido multimedia DASH (codificación, segmentación y generación de descriptores), así como las tareas de análisis e inferencia de emociones a partir de las variables fisiológicas capturadas y la recomendación de contenidos de acuerdo a la emoción inferida según las variables de contexto. En el dominio del proveedor de red se conservan las tareas de distribución del contenido multimedia y gestión de la comunicación extremo a extremo. Finalmente, en el dominio del consumidor se incluyen las tareas de captura de las variables del contexto de usuario, las cuales permiten el análisis e inferencia de emociones. Así mismo, en este dominio se realizan las tareas de estimación del ancho de banda, interpretación de los descriptores asociados a cada contenido multimedia musical y reproducción del contenido. Por lo tanto, en el lado del cliente ante cualquier variación del ancho de banda, se puede acceder a un segmento determinado de contenido multimedia codificado según el estándar DASH. 


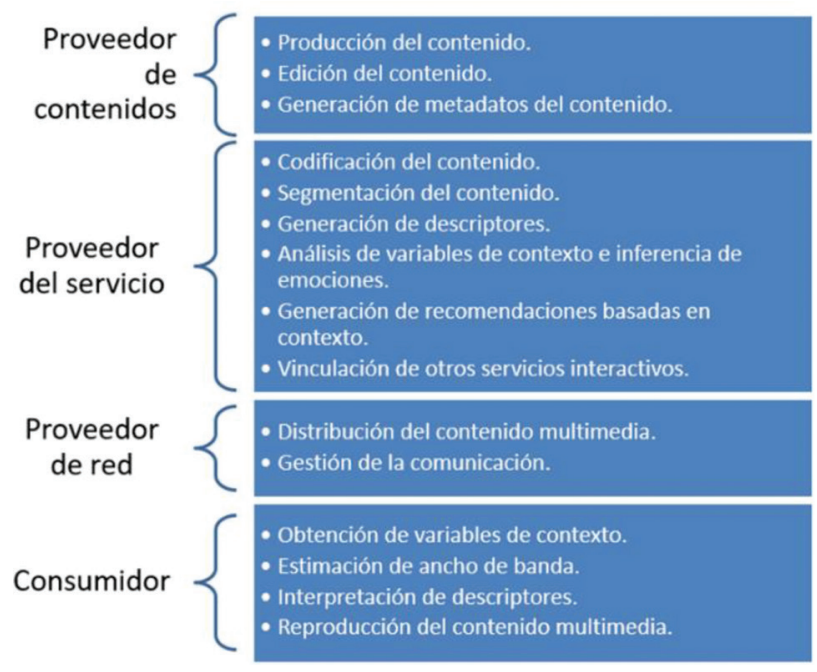

Figura 2 - Vista del negocio

\subsection{Vista del contexto}

Considerando las problemáticas del servicio de VoD, la arquitectura basada en contexto pretende abordar los dos principales retos del servicio, mediante el uso de los componentes arquitectónicos de sistemas de recomendación y streaming DASH, los cuales se enmarcan en las dimensiones del contexto de usuario, red y dispositivo, ver figura 3.

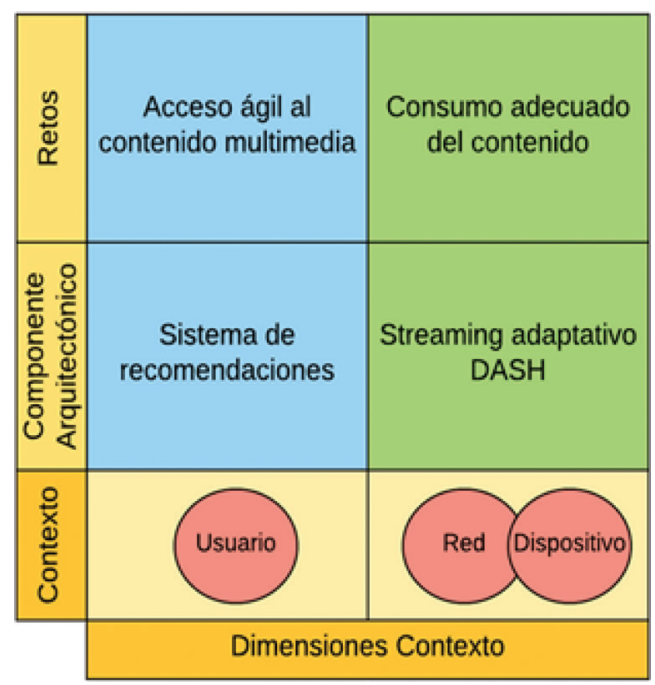

Figura 3 - Vista del negocio 
Mediante el componente de recomendaciones, el cual se enmarca en la dimensión del contexto de usuario, la arquitectura pretende aprovechar variables de tipo fisiológico del usuario, para a partir de ellas inferir una posible emoción de entrada, la cual pueda ser usada para recomendar un conjunto de contenidos musicales previamente clasificados a nivel emocional. Así, se busca mejorar la precisión de las sugerencias de contenidos aportadas por los sistemas de recomendación clásicos, las cuales se centran en las valoraciones de los usuarios, de tal modo que a partir del análisis de variables de tipo fisiológico se puedan generar recomendaciones acordes con las necesidades emocionales de un usuario. Del mismo modo, la vinculación de variables del contexto tiene por objetivo aportar a la solución del problema de arranque en frío. Por su parte, los contenidos multimedia musicales de video se escogieron, teniendo en cuenta la facilidad que tiene la música a través de sus propiedades musicales (arousal, valence, tempo, etc) para ser asociada con las emociones. A pesar de lo anterior, puede usarse cualquier otra aproximación dentro de la computación afectiva que permita clasificar la información de un video (texto, audio, imágenes) dentro de los modelos de emociones convencionales.

Mediante el componente de streaming DASH, el cual se enmarca en las dimensiones del contexto de red y dispositivo, la arquitectura pretende aprovechar las ventajas provistas por DASH, para distribuir contenidos multimedia que se adapten a las características de la red y del dispositivo de acceso. Así, los contenidos multimedia se segmentan, se codifican y describen mediante un archivo MPD en el lado del servidor, de modo que, ante cualquier variación en el ancho de banda detectado en el lado del cliente, se pueda acceder a un determinado segmento del contenido.

\subsection{Vista funcional}

En esta sección se presentan los diferentes componentes funcionales de la arquitectura de VoD, los cuales fueron obtenidos a partir de la vinculación de las dimensiones del

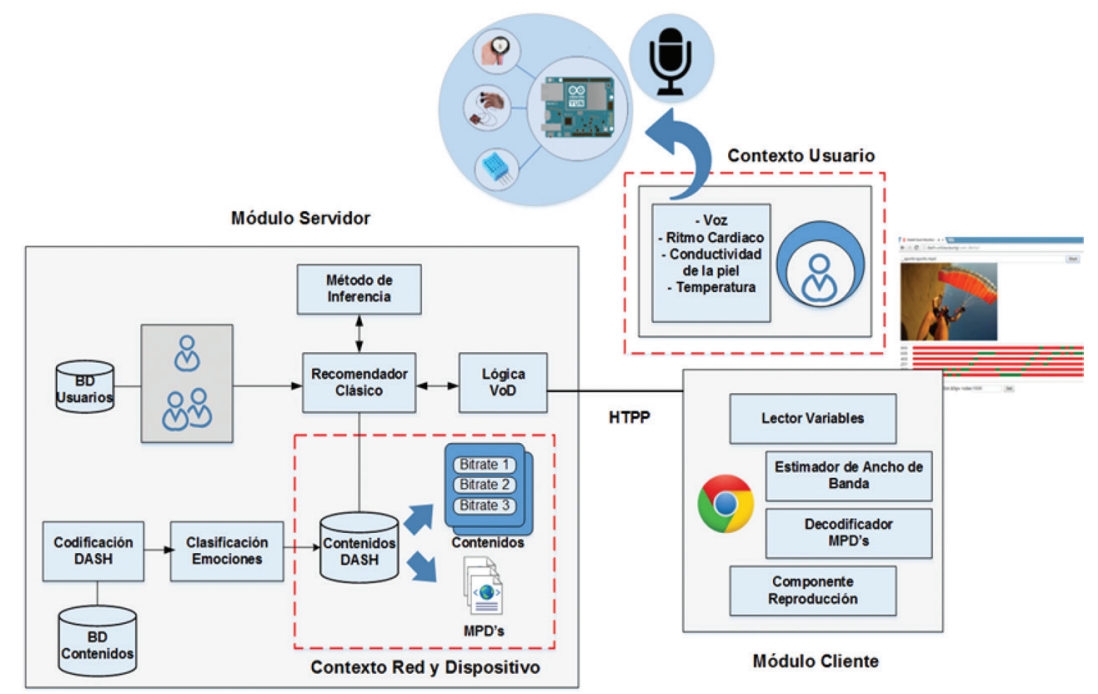

Figura 4 - Vista funcional 
contexto de usuario, dispositivo y red al servicio, ver figura 4. Esta vista funcional de la arquitectura está basada en el modelo cliente-servidor, teniendo en cuenta que la distribución de los contenidos se basa en DASH, es decir que tanto la entrega de los contenidos como la lógica del negocio del servicio son vía HTTP.

El módulo del cliente tiene un sub-módulo denominado: "Lector de variables", el cual tiene asociado a un dispositivo wereable abierto de captura. Esté sub-módulo se encarga de obtener desde el dispositivo cliente, las variables fisiológicas del usuario, las cuales son usadas en el lado del servidor para inferir una emoción de entrada, que a su vez posibilita la recomendación de contenidos musicales de video previamente clasificados según un modelo de emociones. Dentro de las variables de entrada, es posible considerar la voz, la variación de la frecuencia cardiaca, la conductividad de la piel, etc. Adicionalmente, en el lado del cliente se cuenta con el sub-módulo "Estimador de ancho de banda", el cual cumple la función de obtener el ancho de banda disponible en el lado del cliente, para a partir de este acceder al segmento de contenido codificado correspondiente a dicho ancho de banda. La información de los segmentos y su relación con los diferentes anchos de banda, se encuentra consignada en el archivo descriptor MPD de cada contenido, razón por la cual el sub-módulo "Decodificador de MPDs", interpreta estos descriptores, para así obtener el segmento de contenido adecuado para el ancho de banda estimado y proceder con la reproducción del mismo. Adicionalmente, tanto los procesos de captura de variables del contexto y estimación de ancho de banda, se realizan de manera constante con el fin de actualizar el listado de recomendaciones y adaptar el contenido multimedia a los cambios de la red.

El módulo del servidor tiene asociado un sub-módulo denominado: "Lógica VoD”, el cual gestiona la lógica del servicio. Dentro de las funciones de este sub-módulo, se encuentra la recepción de las variables del contexto a partir del cliente, las cuales son enviadas al sub-módulo "Método de Inferencia” para la obtención de una emoción de entrada. Esta emoción de entrada es usada para realimentar las sugerencias generadas por el submódulo "Recomendador Clásico", que a su vez son obtenidas por un clasificador que hace uso del perfil de usuario, así como de las valoraciones y las características musicales de los contenidos multimedia. De este modo, el sub-módulo de "Recomendador Clásico" en conjunto con el sub-módulo "Método de Inferencia" conforman el recomendador basado en contexto.

A partir del listado de sugerencias del recomendador basado en contexto, el usuario puede escoger un contenido multimedia musical de video del catálogo de contenidos. Cada contenido del catálogo ha sido previamente adecuado al formato del estándar DASH y clasificado según un modelo de emociones, por sus características musicales. Para realizar la adecuación del contenido multimedia al formato DASH, se hace uso del submódulo "Codificación DASH”, el cual se encarga de realizar las tareas de codificación, segmentación y generación de descriptores para cada contenido multimedia. En lo que respecta a la clasificación del contenido, el sub-módulo “Clasificación Emociones”, cumple con la función de extraer las características musicales de los contenidos multimedia musicales, para a partir de estas clasificar el contenido según un modelo de emociones. Cabe mencionar, que en el presente artículo se hizo uso de contenidos multimedia musicales, dado que permiten extraer de manera más sencilla las características musicales de arousal y valence, las cuales son usadas para para clasificar el contenido 
multimedia dentro del modelo de emociones propuesto. Finalmente, los contenidos multimedia que pasan el proceso de codificación y clasificación, son dispuestos en un dataset de contenidos.

\subsection{Vista de implementación}

En esta sección se presentan los componentes hardware y software escogidos para la implementación de la arquitectura del servicio de VoD, ver figura 5, los cuales están enmarcados en una arquitectura cliente-servidor. En el módulo del cliente, el usuario tiene adherido a su espacio corporal un conjunto de dispositivos wearables, los cuales permiten la obtención de variables del contexto que son empleadas para inferir una emoción de entrada al sistema, así como realizar un seguimiento sobre los cambios emocionales de un usuario. En el caso de la presente arquitectura, la información del contexto es obtenida mediante la tarjeta compatible con internet de las cosas Arduino Yun, en conjunto con los sensores para la obtención de variables fisiológicas. De igual manera, en el lado del cliente se cuenta con un conjunto de dispositivos de acceso al servicio de VoD. Lo anterior es posible teniendo en cuenta que el servicio ha sido desplegado en un escenario web y cuenta con el soporte del estándar DASH.

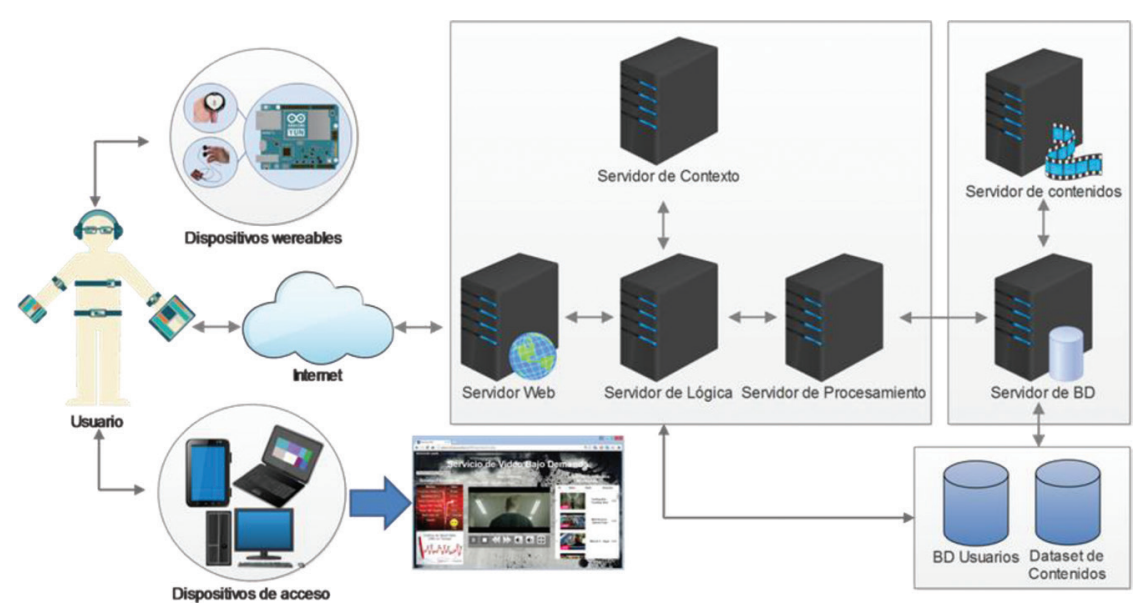

Figura 5 - Vista de implementación

En el módulo del servidor por su parte, se cuenta con un servidor web el cual se encarga de recibir y procesar las peticiones por parte del usuario y enviarlas al servidor de lógica que cumple la función de gestionar la lógica del servicio. Estos dos módulos son componentes conceptuales que están implementados a través de la herramienta (Apache). El servidor de lógica hace uso de un servidor de contexto, el cual se encarga de procesar la información de contexto obtenida en el módulo del cliente, para inferir la emoción percibida por el usuario en un determinado momento. El servidor de contexto fue implementado mediante el framework web Flask, el cual permite el rápido despliegue de servicios en línea mediante el lenguaje Python. De la misma manera, el servidor de 
lógica hace uso de un servidor de procesamiento, el cual cumple con la función de generar el listado de recomendaciones basadas en contexto a partir de la emoción inferida por el servidor de contexto. Para realizar el proceso anterior, el servidor de procesamiento, hace uso del framework web Spark Java y la API de la herramienta de minería de datos Weka. El framework Spark de Java, el cual brinda la facilidad para la construcción y despliegue rápido de servicios de telecomunicaciones. De igual modo, para la generación del listado de recomendaciones, el servidor de procesamiento consulta la base de datos de usuario y el dataset de contenidos multimedia, los cuales son gestionados por el gestor de bases de datos MySQL. El dataset de contenidos multimedia también fue generado en un formato portable como JSON, mediante el uso de la base de datos no-sql TinyDB. Este dataset contiene el listado de contenidos multimedia disponibles y el conjunto de propiedades musicales asociadas a cada uno de ellos, mediante las cuales es posible relacionar un contenido multimedia con una emoción.

Finalmente, el servidor de contenidos multimedia es un componente cuyo propósito es almacenar los contenidos multimedia que son reproducidos en el lado del cliente. Estos contenidos son codificados en diferentes calidades de acuerdo al estándar DASH y tienen asociado un descriptor MPD, con la relación de segmentos de contenido y anchos de banda. Este componente también incluye una herramienta de codificación de contenidos DASH, la cual realiza los procesos de codificación, segmentación y generación del archivo descriptor MPD de manera off-line.

\section{Prototipo del servicio de VoD}

A partir de las diferentes vistas de la arquitectura, en esta sección se presenta un prototipo de servicio de VoD construido a partir de dicha arquitectura, ver figura 6. El prototipo tiene por objetivo proveer un servicio de VoD de contenidos multimedia musicales los cuales al ser consumidos se adaptan a las características de la red y del dispositivo. Así mismo el servicio cuenta con un sistema de recomendaciones cuyas sugerencias son generadas a partir del perfil emocional del usuario, el cual es determinado mediante el seguimiento de variables fisiológicas.

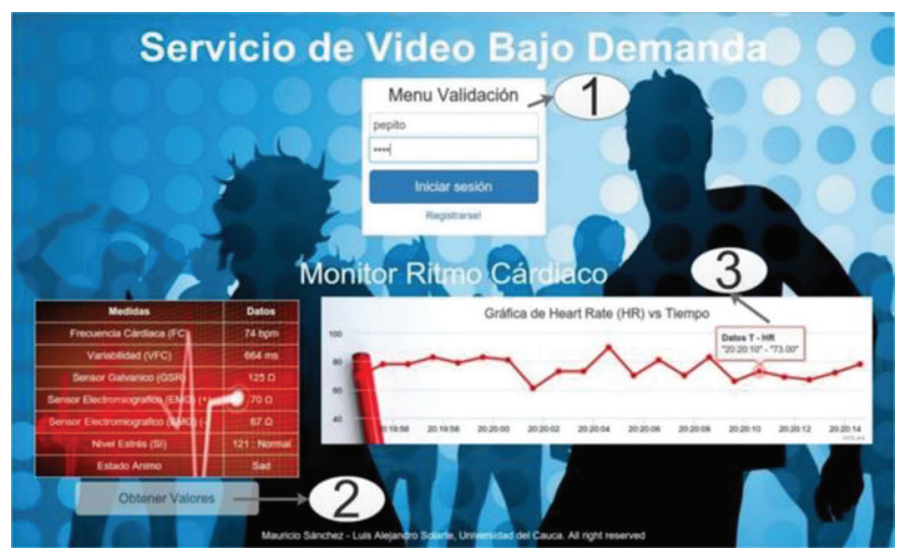

Figura 6 - Validación inicial del servicio 
En primera instancia, en "1" el usuario se valida en el sistema, para ello se obtienen las medidas fisiológicas a través del módulo hardware-software (wereable abierto). En "2", al presionar el botón "Obtener Valores", inicia la captura de la variabilidad de la frecuencia cardiaca VFC y las señales electromiográficas (EMG) del rostro para establecer un nivel de arousal y valence, que a su vez permiten determinar la emoción de entrada del usuario. De igual modo, en "3" el usuario visualiza gráficamente la medida de la frecuencia cardíaca en tiempo real. Una vez obtenidos los valores, el usuario puede iniciar sesión y continuar a la interfaz principal, ver figura 7.

Dentro de la interfaz principal, en "1" el usuario visualiza los videos recomendados según la emoción de entrada, para ello cuenta con el panel de control multimedia y la opción de valorar el contenido. En "2" el usuario puede visualizar el monitoreo continuo de las variables fisiológicas de VFC, EMG y conductividad de la piel (GSR), además se muestra: el nivel de estrés, la emoción que experimenta el usuario segundo a segundo y la gráfica con el seguimiento del ritmo cardiaco. En "3" se presentan al usuario la lista de contenidos recomendados, siendo la emoción detectada por el sistema en cada instante el elemento central para actualizar periódicamente el listado de recomendaciones.

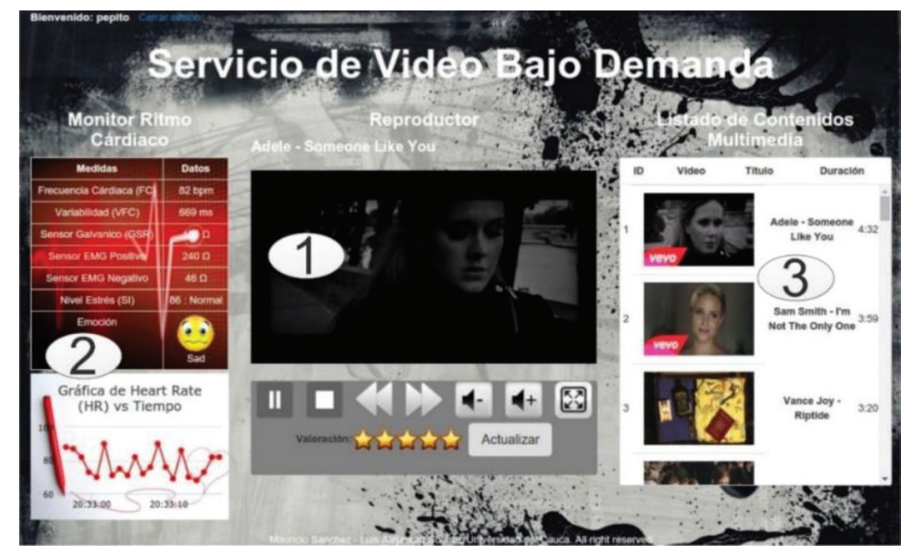

Figura 7 - Interfaz principal del servicio

\section{Conclusiones y trabajos futuros}

En este artículo se presentó una arquitectura para el servicio de VoD desde la vista de negocio, de contexto, funcional y de implementación. Estas vistas ilustran como la definición del contexto para telecomunicaciones puede contribuir a la solución de los dos principales retos del servicio de VoD: acceso ágil y consumo adecuado del contenido multimedia.

La arquitectura propuesta enriquece el servicio de $\mathrm{VoD}$, al abordar diferentes dimensiones de la definición del contexto para servicios de telecomunicaciones, como son: usuario, red y dispositivo, contribuyendo así al mejoramiento de los problemas de acceso ágil y consumo adecuado del contenido multimedia. En la dimensión de usuario la arquitectura considera un conjunto de variables fisiológicas relevantes, mientras que en la dimensión de red y dispositivo la arquitectura está soportada en un escenario de streaming DASH. 
La arquitectura propuesta en este artículo busca aprovechar en la dimensión de usuario la tendencia actual de internet de las cosas, por obtener información de diferentes fuentes conectadas a internet, como es el caso de los sensores integrados en dispositivos wearables. A través del uso de estos dispositivos, se buscó obtener información relevante del usuario, permitiendo la recomendación ágil y precisa de contenidos multimedia según las necesidades del usuario.

La arquitectura para el servicio de VoD aprovecha las emociones asociadas a los contenidos multimedia musicales, de tal modo que permite relacionar el perfil emocional del usuario con un conjunto de contenidos musicales previamente clasificados mediante un sistema de recomendación basado en contexto. Así, a partir de las características musicales de arousal y valence es posible vincular un contenido multimedia con las emociones, las cuales son inferidas usando variables de contexto de usuario.

Como trabajo futuro, se pretende adaptar la arquitectura basada en contexto para la transmisión de servicios de video en vivo, lo cual implica la adecuación del entorno de streaming DASH de la arquitectura, en cuanto a la realización de procesos de codificación y segmentación en tiempo real.

\section{Referencias}

Arzelier, C. (2017). The Mobile Broadband Standard, 3GPP a Global Initiative, Release 12. 3GPP. 3GPP Multimedia Broadcast and Multicast Service (MBMS). Obtenido de: http://www.3gpp.org/ftp/Information/WORK_PLAN/Description_Releases

Adobe. (2012). HTTP Dynamic Streaming. Obtenido de: http://www.adobe.com/ products/hds-dynamic-streaming.html

Asanov, D. (2011). Algorithms and methods in recommender systems. Berlin, Germany: Berlin Institute of Technology. Obtenido de: https://www.snet.tu-berlin.de/ fileadmin/fg220/courses/SS11/snet-project/recommender-systems_asanov.pdf

Campo, W., Arciniegas, J., Garcia, R., \& Melendi, D. (2010). Análisis de Tráfico para un Servicio de Vídeo bajo Demanda sobre Recles HFC usando el Protocolo RTMP. Información tecnológica, 21(6), 27-48.

Casín, B. (2013). DASH: Un estándar MPEG para streaming sobre HTTP (tesis de pregrado). Barcelona: Universitat Politecnica de Catalunya.

Chanchí, G. E., Campo, W., Amaya, J., \& Arciniegas, J. (2011). Esquema de Servicios para Televisión Digital Interactiva, basados en el protocolo REST-JSON. Gramado, Brasil: Cadernos de Informatica.

Chen, G., \& Kotz, D. (2000). A survey of context-aware mobile computing research (reporte técnico). Hanover, NH, USA: Dartmouth College. Obtenido de: https://dl.acm.org/citation.cfm?id=867843.

Cunningham, S., Caulder, S., \& Grout, V. (2008). Saturday Night or Fever? ContextAware Music Playlists. Proceedings of the 3rd International Audio Mostly conference on Sound in Motion. 
da Silva, F., Alves, L., \& Bressan, G. (2009). PersonalTVware: A Proposal of Architecture to Support the Context-aware Personalized Recommendation of TV Programs. In Proc. of 7 th Eur. Conf. Interactive TV and Video. Leuven-Belgica.

Dabrowski, M., Gromada, J., \& Moustafa, H. (2012). Context-Awareness for IPTV Services Personalization. In 2012 Sixth International Conference on Innovative Mobile and Internet Services in Ubiquitous Computing (pp. 37-44). Obtenido de: https://doi.org/10.1109/IMIS.2012.97

Gambín, J. (2012). Desarrollo de un servicio de televisión interactiva HbbTV según el estándar ETSI TS 102796 v1.1.1. (Tesis de pregrado). Cartagena-España: Universida Politécnica de Cartagena.

ISO/IEC (2014). ISO/IEC 23009-1:2014. (2014). Information technology - Dynamic adaptive streaming over HTTP (DASH) - Part 1: Media presentation description and segment formats. Geneva, Switzerland: International Organization for Standardization.

ITU-T.(2008).ITU-TY.1900-series-Supplementon IPTVserviceusecases. International Telecommunication Union. Series Y: Global information Infrastructure, Internet Protocol Aspects and Next-Generation Networks. Obtenido de https://www.itu.int/rec/T-REC-Y.Sup5-200805-I/

Jannach, D., Zanker, M., Felfernig, A., \& Friedrich, G. (2010). Recommender Systems: An Introduction. Cambridge.

Kruchten, P. (1995). Architectural Blueprints-The “4+1" View Model of Software Architecture. IEEE Software, 12(6), 42-50.

Li, Z., \& Bouazizi, I. (2013). Light Weight Content Fingerprinting for Video Playback Verification in MPEG DASH. 2oth International Packet Video Workshop. San Jose - USA: IEEE.

Mack, S. (2002). Streaming Media Bible. Hoboken, NJ: John Wiley \& Sons.

Melville, P., Mooney, R. J., \& Nagarajan, R. (2002). Content-Boosted Collaborative Filtering for Improved Recommendations. XVIII Conferencia Internacional de Inteligencia Artificial - AAAI 20O2. Edmonton, Canadá.

Muller, C., Lederer, S., Rainer, B., Waltl, M., Grafl, M., \& Timmerer, C. (2013). Open source column: dynamic adaptive streaming over HTTP toolset. ACM SIGMultimedia Records, 5(1), 11-13. Obtenido de https://doi.org/10.1145/2484912.2484915

Ortiz, D., Chanchí, G., Arciniegas, J., \& Duran, D. (2017). Escenario para la transmisión de streaming adaptativo DASH-WebM. Revista Colombiana de Computación $R C C, 18(1), 27-45$.

Park, H., Yoo, J., \& Sung-Bae, C. (2006). A Context-Aware Music Recommendation System Using Fuzzy Bayesian Networks with Utility Theory. Fuzzy Systems and Knowledge Discovery, 4223, 970-979. 
Porcel, C., López-Herrera, A., \& Herrera, E. (2009). A recommender system for research resources based on fuzzy linguistic modeling. Expert Systems with Applications, $36(3), 5173-5183$.

Porcel, C., Moreno, J. M., \& Herrera, E. (2009). A multi-disciplinar recommender system to advice research resources in University Digital Libraries. Expert Systems with Applications: An Internacional Journal, 36(10), 12520-12528.

Porcel, C., Tejeda, A., Martínez, M., \& Herrera, E. (2012). A hybrid recommender system for the selective dissemination of research resources in a Technology Transfer Office. Information Sciences, 184(1), 1-19.

Pripuzic, K., Zarko, I., Podobnik, V., Lovrek, I., Cavka, M., Petkovic, I., Stulić, P., \& Gojčeta, M. (2013). Building an IPTV VoD Recommender System: An Experience Report.In Proceedings of the 12th International Conference on Telecommunications: ConTEL 2013: Zagreb, Croatia, June 26-28, 2013. University of Zagreb, Faculty of Electrical Engineering and Computing. Obtenido de https://ieeexplore.ieee.org/document/6578284

Rho, S., Han, B., \& Hwang, E. (2009). SVR-based music mood classification and contextbased music recommendation. In Proceedings of the seventeen ACM international conference on Multimedia - MM 'og (p. 713). New York, New York, USA: ACM Press. Obtenido de https://doi.org/10.1145/1631272.1631395

Song, S., Moustafa, H., \& Afifi, H. (2011). IPTV Services Personalization Using ContextAwareness. Informatica, 13-20.

Su, J., Yeh, H., Yu, P., \& Tseng, V. (2010). Music Recommendation Using Content and Context Information Mining. Intelligent Systems, 25(1), 16-26.

Thawani, A., Gopalan, S., \& Sridhar, V. (2004). Context Aware Personalized Ad Insertion in an Interactive TV Environment. In Proceedings of Workshop on Personalization in Future TV.

Timmerer, C., \& Griwodz, C. (2012). Dynamic Adaptive Streaming Over HTTP - From Content Creation to Consumption. In Proceedings of the 2oth ACM international conference on Multimedia - MM '12 (p. 1533). New York, New York, USA: ACM Press. Obtenido de https://doi.org/10.1145/2393347.2396553

Turrin, R., \& Cremonesis, P. (2010). Recomender Systems for Interactive TV. In Proceedings of the 8th European Conference on Interactive TV and Video conference. June 9-11, 2010, Tampere, Finland. Obtenido de https://dl.acm.org/citation.cfm?id=1809777

Wang, X., Rosenblum, D., \& Wang, Y. (2012). Context-aware mobile music recommendation for daily activities. In Proceedings of the 2Oth ACM international conference on Multimedia - MM'12 (p. 99). New York, New York, USA: ACM Press. Obtenido de: https://doi.org/10.1145/2393347.2393368

Yager, R. (2003). Fuzzy logic methods in recommender systems. Fuzzy Sets and Systems, 136(2), 133-149. 
Zambelli, A., (2009). IIS Smooth Streaming Technical Overview. Obtenido de http:// www.bogotobogo.com/VideoStreaming/Files/iis8/IIS_Smooth_Streaming_ Technical_Overview.pdf

Zanker, M., Klagenfurt, U., Jannach, D., Gordea, S., \& Jessenitschnig, M. (2007). Comparing Recommendation Strategies in a Commercial Context. IEEE Intelligent Systems, 22(3), 69-73. Obtenido de: https://doi.org/10.1109/MIS.2007.49

Zhang, H., \& Zheng, S. (2005). Personalized TV program recommendation based on TV-anytime metadata. In Proceedings of the Ninth International Symposium on Consumer Electronics, 2005. (ISCE 2005). (pp. 242-246). Obtenido de: https://doi.org/10.1109/ISCE.2005.1502378. 